\title{
Indicadores de gravidade da depressão materna: impacto para problemas comportamentais de escolares*
}

\section{Severity indicators of maternal depression: impact on behavioral problems of schoolchildren \\ Indicadores de gravedad de la depresión materna: impacto en los problemas de conducta de los escolares}

\author{
Recebido: $18 / 10 / 2019$ \\ Aprovado: 05/04/2020 \\ Publicado: 01/08/2020
}

\section{Fernanda Aguiar Pizeta ${ }^{1}$ Ana Paula Casagrande Silva-Rodrigues ${ }^{2}$ Sonia Regina Loureiro ${ }^{3}$}

Este é um estudo transversal realizado na cidade de Ribeirão Preto, que tem como objetivo sistematizar indicadores clínicos associados à gravidade da depressão recorrente em mães de crianças em idade escolar e avaliar o impacto desses indicadores para o comportamento dos filhos, bem como, identificar se há interferência de determinantes sociais associados às condições sociodemográficas das famílias. A coleta de dados considerou o período de 2007 a 2013, avaliando-se 100 mães com filhos em idade escolar, divididos em Grupo Depressão e Grupo Comparação com 50 díades cada. Utilizou-se Questionário sociodemográfico, Questionário sobre a Saúde do Paciente-9, Entrevista Clínica Estruturada e, Questionário de Capacidades e Dificuldades. Verificou-se que, independente de vulnerabilidades sociais, a gravidade da depressão se configurou como fator de risco para mais problemas de comportamento das crianças no geral $\left(p<0,001 ; R^{2} a=0,138\right)$, internalizantes (sintomas emocionais - $p<0,001 ; R^{2} a=0,186$ - e problemas de relacionamento - $p<0,001 ; R^{2} a=0,141$ ) e externalizantes (problemas de conduta - $\mathrm{p}=0,044 ; \mathrm{R}^{2} \mathrm{a}=0,031$ ). A avaliação de indicadores clínicos específicos da depressão pode ser utilizada como complemento no rastreamento sistemático da depressão em diferentes níveis de atenção em saúde, ampliando a qualidade das avaliações e os cuidados à saúde mental materna-infantil.

Descritores: Depressão; Criança; Comportamento; Saúde mental; Índice de gravidade de doença.

This is a cross-sectional study conducted in the city of Ribeirão Preto, SP, Brazil, aims to systematize clinical indicators associated with the severity of recurrent depression in mothers of school-aged children and assess the impact of these indicators on their children's behavior, as well as to identify whether there is interference of social determinants associated with the sociodemographic conditions of families. Data collection considered the period from 2007 to 2013, 100 mothers with school-age children were evaluated and divided into Depression Group and Comparison Group with 50 dyads each. Sociodemographic questionnaire, Questionnaire on Patient Health-9, Structured Clinical Interview and Capacity and Difficulties Questionnaire were used. It was found that, regardless of social vulnerabilities, the severity of depression was configured as a risk factor for more behavioral problems in children in general $\left(\mathrm{p}<0.001 ; \mathrm{R}^{2} \mathrm{a}=0.138\right)$, internalizing (emotional symptoms $-\mathrm{p}<0.001 ; \mathrm{R}^{2} \mathrm{a}=0.186-$ and relationship problems $-p<0.001 ; R^{2} a=0.141$ ) and externalizing (conduct problems $-p=0.044 ; R^{2} a=0.031$ ). The evaluation of specific clinical indicators of depression can be used as a complement in the systematic screening of depression at different levels of health care, expanding the quality of evaluations and the care for maternal and child mental health.

Descriptors: Depression; Child; Behavior; Mental health; Severity of ilness index.

Este es un estudio transversal realizado en la ciudad de Ribeirão Preto, SP, Brasil, que tiene como objetivo sistematizar los indicadores clínicos asociados a la gravedad de la depresión recurrente en madres de niños en edad escolar y evaluar el impacto de estos indicadores en el comportamiento de sus hijos, así como identificar la interferencia de determinantes sociales asociados a las condiciones sociodemográficas de las familias. La recopilación de datos consideró el período de 2007 a 2013, evaluando a 100 madres con hijos en edad escolar, divididos en Grupo Depresión y Grupo Comparación con 50 díadas cada una. Se utilizaron Cuestionario sociodemográfico, Cuestionario sobre la salud del Paciente-9, Entrevista Clínica Estructurada y Cuestionario de Capacidad y Dificultades. Se verificó que, independientemente de las vulnerabilidades sociales, la gravedad de la depresión se configuró como un factor de riesgo para más problemas de comportamiento de los niños en general $\left(\mathrm{p}<0.001 ; \mathrm{R}^{2} \mathrm{a}=0.138\right)$, internalizantes (síntomas emocionales - $\mathrm{p}<0.001 ; \mathrm{R}^{2} \mathrm{a}=0.186$ - y problemas de relación $\mathrm{p}<0.001 ; \mathrm{R}^{2} \mathrm{a}=0.141$ ) y externalizantes (problemas de conducta $-\mathrm{p}=0.044 ; \mathrm{R}^{2} \mathrm{a}=0.031$ ). La evaluación de indicadores clínicos específicos de la depresión se puede utilizar como un complemento en la detección sistemática de la depresión en diferentes niveles de atención médica, ampliando la calidad de las evaluaciones y la atención de la salud mental materna e infantil.

Descriptores: Depresión; Niño; Conducta; Salud mental; Índice de severidad de la enfermedad.

\footnotetext{
* Trabalho financiado pelo Conselho Nacional de Desenvolvimento Tecnológico e Científico (CNPq).

1. Psicóloga. Especialista em Violência Doméstica. Mestre e Doutora em Ciências. Pós-Doutora em Psicologia. Docente da Universidade Paulista - Campus Ribeirão Preto e, Psicóloga Judiciário do Tribunal de Justiça do Estado de São Paulo, Ribeirão Preto, SP, Brasil. ORCID 0000-0002-9864-1054 E-mail: fepizeta@gmail.com 2. Psicóloga. Especialista em Psicologia Hospitalar. Mestre e Doutora em Ciências. Psicóloga do Hospital das Clínicas da Faculdade de Medicina de Ribeirão Preto da Universidade de São Paulo (FMRP-USP), Ribeirão Preto, SP, Brasil. ORCID: 0000-0001-9672-5118 E-mail: anapaulacasagrande@usp.br

3. Psicóloga. Especialista em Psicologia Clínica. Mestre e Doutora em Psicóloga Clínica. Docente da FMRP-USP, Ribeirão Preto, SP, Brasil. ORCID:0000-0001-9423-2897 E-mail: srlourei@fmrp.usp.br
} 


\section{INTRODUÇÃO}

$\mathbf{0}$ transtorno depressivo se destaca dentre os transtornos mentais pela incidência e recorrência, configurando-se em uma vivência de sofrimento ou prejuízo clinicamente significativo com impacto no funcionamento em diferentes áreas da vida do indivíduo, com alta mortalidade associada especialmente ao suicídio ${ }^{1}$.

Dados da Organização Mundial de Saúde (OMS) destacam que a depressão é o transtorno que, sozinho, mais contribui para desfechos disfuncionais, tendo uma prevalência estimada de 4,4\% da população mundial ${ }^{2}$ e um decorrente número de mortes por suicídio em cerca de 800 mil pessoas por ano no mundo ${ }^{3}$. A incidência da depressão é maior em mulheres $(5,1 \%) \mathrm{em}$ comparação aos homens $(3,6 \%)^{2}$. Essa psicopatologia chega a ser até três vezes mais frequente nas mulheres, especialmente naquelas com idade entre 18 e 29 anos $^{1}$, ou seja, em idade fértil.

A relevância do estudo da depressão em mulheres que são mães relaciona-se à prevalência do transtorno especificamente frente a tal condição, que demanda atenção em saúde mental, inclusive em unidades básicas de saúde (UBS). Os casos de depressão não estão sendo cuidados apenas nos equipamentos secundários de saúde mental, mas também na atenção primária, sendo que um quarto da demanda relacionada a esse transtorno estaria sendo acompanhado nas UBS no Brasil ${ }^{4}$, o que evidencia a relevância de ações de saúde mental nos diversos serviços.

Considerando-se a característica de recorrência do transtorno depressivo, a maior vulnerabilidade das mulheres, especialmente em idade fértil, pondera-se que o funcionamento afetivo disfuncional associado ao transtorno tem impacto não só para a pessoa acometida, mas também para as pessoas que convivem com ela. No caso das mulheres/mães é relevante considerar as possíveis influências da depressão materna para o desenvolvimento dos filhos.

Conviver com a depressão materna pode acarretar prejuízos socioemocionais ${ }^{5}$ para a criança em idade escolar. Os estudos mostram o predomínio de mais sintomas relativos aos comportamentos internalizantes ${ }^{6,7}$, bem como sintomas internalizantes e externalizantes ${ }^{8,9}$, além de prejuízos para a habilidade cognitiva ${ }^{9}$ e para as habilidades sociais ${ }^{10}$.

0 impacto da depressão se amplia, de maneira geral, tendo em vista sua associação com outras condições de vulnerabilidade, que evidenciam um cenário familiar e social também disfuncional. Dessa forma, faz-se relevante que essa psicopatologia seja estudada em associação a determinantes sociais que concorrem para esse transtorno.

Segundo a $\mathrm{OMS}^{11}$, os prejuízos vivenciados pela mulher/mãe, seus filhos e sua família tendem a se relacionar à baixa escolaridade das mães, menor renda, piores condições materiais e econômicas da família, além de menor suporte social. Estudos empíricos e de revisão da literatura ${ }^{12-14}$ que avaliam o impacto da depressão materna e de condições cumulativas de risco para o desenvolvimento infantil sustentam a compreensão da OMS e ampliam o cenário de seu desenvolvimento para incluir fatores internos à família, relativos à qualidade das interações e dos vínculos estabelecidos entre os pais e os filhos ${ }^{12}$ e na conjugalidade, tal como a monoparentalidade ${ }^{13}$ e os conflitos conjugais ${ }^{14}$.

O reconhecimento do impacto da depressão para o contexto familiar e de desenvolvimento dos filhos sinaliza a necessidade de que se amplie a compreensão quanto às manifestações clínicas e evolutivas desse transtorno. Destaca-se a importância de examinar a gravidade dos sintomas frente à condição de recorrência, e seu impacto para os desfechos desenvolvimentais dos filhos.

Os episódios depressivos são caracterizados por um conjunto de sintomas persistentes, associados ao humor entristecido ou irritadiço, apatia e perda de interesse/prazer, bem como a outros prejuízos cognitivos e a alterações somáticas, que tendem a se repetir na maioria dos $\operatorname{casos}^{1}$. 0 curso desse transtorno pode ser variável, podendo ocorrer períodos de remissão dos sintomas entre os episódios depressivos ou aumento na gravidade dos sintomas conforme o número de ocorrências dos episódios. As baixas taxas de recuperação de um episódio, condição que favoreceria a diminuição significativa da sintomatologia e a disfuncionalidade, estão 
associadas ao número de episódios anteriores, vivências psicóticas e outros indicadores de gravidade ${ }^{1}$.

Destaca-se que quanto maior a gravidade do transtorno, maior o prejuízo funcional e sofrimento psíquico decorrente, sendo estes indicadores que direcionam o tratamento a ser oferecido à pessoa acometida pelo transtorno. Cada episódio depressivo pode ser avaliado em função de sua gravidade, variando entre leve, moderada e grave, de acordo com o número de sintomas diagnosticados e nível de prejuízo funcional desencadeado. A maior gravidade dos episódios depressivos se associa ao maior número de sintomas, à intensidade desses sintomas e ao sofrimento desencadeado, que tende a não ser manejável pela própria pessoa, e à interferência desses sintomas de forma acentuada no funcionamento social e profissional da pessoa ${ }^{1}$.

0 presente estudo tem como objetivo sistematizar indicadores clínicos associados à gravidade da depressão recorrente em mães de crianças em idade escolar e avaliar o impacto desses indicadores para o comportamento dos filhos, bem como, identificar se há interferência de determinantes sociais associados às condições sociodemográficas das famílias.

\section{MÉTODO}

Este é um estudo com delineamento transversal, correlacional preditivo, desenvolvido na cidade de Ribeirão Preto e, apreciado e aprovado segundo o Processo CEP-HCFMRP no 6395/2011. Adotaram-se os cuidados éticos preconizados pela Declaração de Helsinki e normativas brasileiras referentes à pesquisa com seres humanos, garantindo-se sobremaneira a voluntariedade dos participantes e a assinatura do Termo de Consentimento Livre e Esclarecido (TCLE) para início da coleta de dados.

Participaram do presente estudo, díades mãe-criança, de uma cidade de médio porte do interior do Estado de São Paulo, distribuídas em dois grupos:

Grupo Depressão: díades mãe-criança, cujas mães apresentaram história de depressão recorrente, com pelo menos um episódio moderado ou grave nos dois anos anteriores e sem sintomas nos seis meses prévios, atendidas em serviços ambulatoriais públicos de saúde mental, adotando-se a Classificação Internacional de Doenças e Problemas Relacionados à Saúde - CID-1015;

Grupo Comparação: díades mãe-criança, cujas mães não apresentaram história de depressão ou qualquer transtorno psiquiátrico, sendo identificadas junto a uma Unidade de Saúde da Família da Secretaria Municipal de Saúde.

Foram excluídos casos do Grupo Depressão nos quais a mulher apresentava diagnósticos de depressão maior com episódio único e exclusivamente com episódios leves nos últimos dois anos; mães que apresentaram episódios moderados ou graves nos últimos seis meses e que apresentaram comorbidades com outros transtornos psiquiátricos.

Além disso, excluíram-se mães e crianças com deficiências aparentes e/ou sintomas atuais ou histórico de outras doenças crônicas graves, tais como: câncer, HIV/AIDS, cardiopatia, nefropatia, hepatopatia, hanseníase, esclerose múltipla, Parkinson, tuberculose ativa e paralisia irreversível e incapacitante. Essas doenças foram elencadas com base no artigo 151 da Lei 8.213/91 (Planos de Benefícios da Previdência Social), que dispõe de uma lista de doenças consideradas graves e incapacitantes e doenças crônicas não transmissíveis no Brasil ${ }^{16}$.

A identificação das mulheres se deu através dos prontuários dos serviços de saúde mental para o Grupo Depressão e, no Grupo Comparação, captou-se lista pelo serviço de saúde.

Quanto aos instrumentos empregados, incluíram-se indicadores relativos à saúde mental das mães, condições sociodemográficas das famílias e problemas de comportamento dos filhos, mediante aplicação junto às mulheres dos seguintes instrumentos:

Questionário geral: elaborado para o estudo, permitindo o levantamento de características sociodemográficas dos participantes e de suas famílias, a saber: idades da mãe e da criança; estado civil da mãe; escolaridade da mãe, do pai e da criança; profissão/ocupação 
materna e paterna; composição familiar; condições de moradia; renda familiar; e nível socioeconômico. Tal Questionário avaliou também condições da gravidez, histórico de depressão familiar, de violência doméstica, presença de doenças crônicas e de deficiências por parte das mães e das crianças.

Questionário sobre a Saúde do Paciente-9 (PHQ-9): instrumento de rastreamento de sintomas depressivos atuais, validado por Spitzer, Kroenke e Williams ${ }^{17}$ e Kroenke, Spitzer e Williams ${ }^{18}$, com bons indicadores psicométricos na realidade brasileira ${ }^{19}$. Esse questionário é composto por nove itens respondidos frente à presença e frequência de sinais e sintomas de depressão nas últimas duas semanas, podendo o escore total variar de zero a 27. Considerouse como indicador positivo de sinais e sintomas de Depressão Maior valores maiores ou iguais a 1017,18, sendo respondido pelas mães do Grupo Comparação de forma a serem excluídas as mulheres com indicador positivo.

Entrevista Clínica Estruturada para o DSM-IV (SCID): entrevista semiestruturada para avaliação diagnóstica, traduzida e adaptada para população brasileira por Del-Ben ${ }^{20}$ e Del-Ben e cols ${ }^{21}$. Foram utilizados para o Grupo Depressão a Revisão Geral, a SCID I (módulos A, B, C, D, E e F) e a SCID II, visando à aplicação dos critérios de inclusão das mães, ou seja, a confirmação diagnóstica de transtorno depressivo recorrente e a exclusão de comorbidades. Além das informações diagnósticas, a SCID possibilitou a coleta de informações sobre o perfil clínico desse grupo, a saber: gravidade dos episódios depressivos, número de recorrência de tais episódios, número de internações, presença de sintomas psicóticos, ideação suicida, tentativa de suicídio, uso atual de antidepressivo e os períodos de ocorrência dos episódios depressivos, focalizando de modo especial o período de exposição da criança à depressão materna. As mães do Grupo Comparação responderam a versão SCID-NP (edição para não pacientes), a fim de identificar aquelas com transtornos psiquiátricos passados ou atuais, as quais foram excluídas do estudo.

Questionário de Capacidades e Dificuldades (SDQ): instrumento de uso livre (site: www.sdqinfo.com), desenvolvido por Goodman ${ }^{22}$ para rastreamento de problemas comportamentais em crianças e adolescentes (de quatro a 16 anos) nos últimos seis meses. Este Questionário foi traduzido e adaptado para a realidade brasileira por Fleitlich, Cortázar e Goodman ${ }^{23}$ e apresenta três versões de aplicação (pais, professores e jovens a partir dos 11 anos), sendo que no presente estudo se utilizou a versão para pais. 0 supracitado Questionário é composto por 25 itens, os quais são divididos em quatro escalas de dificuldades (sintomas emocionais, problemas de conduta, hiperatividade e problemas de relacionamento com os colegas) e uma escala de capacidade (comportamento pró-social) com cinco itens cada. Cada item apresenta três alternativas de resposta, sendo elas: verdadeiro, mais ou menos verdadeiro e falso, cuja pontuação varia entre zero, um e dois. A pontuação máxima, em cada uma das escalas, é de 10 pontos. 0 escore total de dificuldades é obtido pela somatória da pontuação nas quatro escalas de dificuldades, variando de zero a 40. Quanto maior a pontuação nas escalas de dificuldades ou no escore total, mais problemas comportamentais na área específica avaliada ou no geral aparecem respectivamente. Para escala de capacidade, quanto maior a pontuação obtida, mais recursos pró-sociais a criança apresenta. A pontuação em cada escala e no escore total de dificuldades possibilita também a classificação em três categorias: normal, limítrofe e anormal. Apresenta bons indicadores psicométricos ${ }^{24}$.

A coleta com as díades de ambos os grupos (Depressão e Comparação) foi realizada, em um único momento, entre os anos de 2007 e 2013, nos serviços de saúde em que as mães eram atendidas ou em suas casas, respeitando-se a preferência das mesmas. Buscou-se garantir a privacidade e o sigilo em todos os locais mencionados. Todas as mães que atenderam aos critérios de seleção e aceitaram participar do estudo foram contatadas pessoalmente, em horário previamente agendado, sendo ressaltado o caráter voluntário da participação e a possibilidade de desistência a qualquer momento, sem prejuízos para si ou para seus filhos. 
O início das avaliações ocorreu somente após a leitura e assinatura pelas mães do TCLE. As avaliações foram realizadas em sessões individuais, face a face, por pesquisadores previamente treinados, visando tornar homogênea a coleta de dados. As mães responderam ao Questionário geral, à Entrevista semiestruturada, ao PHQ-9 no caso das mães do Grupo Comparação, e ao SDQ.

Os dados relativos às características sociodemográficas foram analisados descritivamente, comparando-se o Grupo Depressão e o Grupo Comparação mediante Teste do Qui-Quadrado ou Teste Exato de Fisher (variáveis categóricas) e Teste $t$ de Student (variáveis contínuas). Para tomada de decisão quanto aos testes adotados, procedeu-se ao teste de normalidade Komolgorov-Smirnov. As análises estatísticas foram realizadas por meio do software IBM SPSS Statistic, versão 24 para Windows.

A gravidade da depressão materna pautou-se em dados provenientes da SCID ao se realizar o diagnóstico de depressão, o qual se caracteriza pelos critérios estabelecidos pelo DSM. Dessa forma, elencaram-se sete indicadores que se associam teórica e clinicamente à gravidade do Transtorno Depressivo Recorrente, atribuindo-se peso para cada um deles. Construiu-se uma breve escala de itens que variaram entre zero e dois pontos, sendo que a somatória da pontuação nos sete itens permitiu a identificação de um escore total.

Tendo em vista a possibilidade de itens cumulativos, escores maiores indicam a presença de maior gravidade do transtorno em questão, considerando-se assim a depressão enquanto variável escalar. 0 Quadro 1 apresenta o rol de variáveis, os critérios utilizados e a pontuação atribuída para cada critério, compondo uma escala em que indicadores cumulativos de gravidade foram contemplados.

Quadro 1. Critérios utilizados para codificação e pontuação de cada variável incluída, relativa ao perfil clínico das mães com depressão recorrente.

\begin{tabular}{|l|l|c|}
\hline Variáveis & Critério utilizado & Pontuação \\
\hline \multirow{2}{*}{ Gravidade do Último Episódio } & Moderado & 1 \\
\cline { 2 - 3 } & Grave & 2 \\
\hline \multirow{2}{*}{ Sintomas Psicóticos* } & Ausente & 0 \\
\cline { 2 - 3 } & Presente & 1 \\
\hline \multirow{2}{*}{ Internações Psiquiátricas* } & Ausente & 0 \\
\cline { 2 - 3 } & Presente & 1 \\
\hline \multirow{2}{*}{ Ideação suicida } & Ausente & 0 \\
\cline { 2 - 3 } Tentativa de suicídio* & Presente & 1 \\
\hline \multirow{2}{*}{ Número de recorrências* } & Ausente & 1 \\
\cline { 2 - 3 } & Presente & 0 \\
\hline \multirow{2}{*}{ Percepção materna da situação atual } & Duas crises & 1 \\
\cline { 2 - 3 } & Três ou mais crises & 0 \\
\cline { 2 - 3 } & Positiva e Positiva com recaídas & 1 \\
\hline \multicolumn{2}{|c|}{ Tositiva ou Negativa com prejuízos (Pontuação Máxima = 8) } & \\
\hline
\end{tabular}

Nota: *Refere-se ao indicador avaliado ao longo da história do Transtorno Depressivo Recorrente

Tais itens subsidiaram a avaliação da gravidade da depressão materna, a partir da gravidade do último episódio depressivo (moderado ou grave), presença de sintomas psicóticos, de internações psiquiátricas, de ideação e tentativa de suicídio, número de recorrências desses episódios (dois, três ou mais de três episódios bem definidos), e percepção materna referente a sua condição atual de saúde mental (positiva, com recaídas, negativa e com prejuízos).

A partir dos escores associados à gravidade da depressão, procedeu-se às análises de predição. Realizou-se análise de regressão linear univariada ${ }^{25}$ para verificar o efeito da depressão materna para os problemas de comportamento de escolares, avaliado pelo escore total de dificuldades e escores nas quatro subescalas de problemas do SDQ. Em tais análises, considerou-se o escore relativo à soma dos indicadores de gravidade da depressão. Para dirimir eventuais pesos das variáveis sociodemográficas, realizou-se análise de regressão logística 
bivariada $^{25}$ para informações quanto ao efeito preditor de tais variáveis isoladas para problemas de comportamento das crianças. Adotou-se nível de significância de $5 \%(p \geq 0,05)$.

\section{RESULTADOS}

Participaram 100 díades de mães com seus filhos, sendo 50 no Grupo Depressão e 50 no Grupo Comparação. As mães tinham entre 25 e 45 anos de idade. As crianças de ambos os sexos, estavam na faixa etária de sete e 12 anos (idade escolar) e conviviam com a mãe biológica.

As crianças apresentaram média de idade 9,9 anos (variação de sete anos e três meses a 12 anos e nove meses; DP $=1,68$ ), as quais foram distribuídas homogeneamente entre as faixas etárias e gênero para ambos os grupos. Quanto à escolaridade, todas as crianças frequentavam o Ensino Fundamental, sendo que a maioria (61\%) estava nos anos iniciais (1음 ao ${ }^{\circ}$ ano), com distribuição semelhante para ambos os grupos. Não foram identificadas diferenças estatisticamente significativas para as variáveis sociodemográficas das crianças, o que evidenciou distribuição equilibrada entre os grupos, conforme os critérios de inclusão do estudo (Tabela 1).

As mães tinham idade média de 36,3 anos (variação entre 25 e 45 anos, DP=5,04). Verificou-se uma distribuição homogênea, sendo a média de idade das mães do Grupo Depressão de 36,6 anos (DP=5,16) e a do Grupo Comparação de 35,9 anos (DP=4,94). Em relação à ocupação, notou-se o predomínio de mães que referiram exercer alguma atividade profissional remunerada em ambos os grupos (Depressão - 54\% e Comparação - 72\%). A maioria (87\%) das mães tinha de um a três filhos, variando de um a cinco filhos, embora $20 \%$ das mães do Grupo Depressão e 6\% do Grupo Comparação terem quatro a cinco filhos. Para essas variáveis, não se detectou diferença estatisticamente significativa entre os grupos (Tabela 1).

Para as demais variáveis sociodemográficas maternas, foram identificadas diferenças estatisticamente significativas entre os grupos Depressão e Comparação. A maioria das mães (56\%) referiram ter mais de oito anos de estudo, sendo que 62\% das mães do Grupo Depressão tinham até oito anos de estudo e 74\% das mães do Grupo Comparação mais de oito anos. Em relação ao estado civil, 66\% das mães do Grupo Depressão afirmaram estar sem companheiro (solteiras, separadas, desquitadas ou viúvas), sendo cuidadoras exclusivas de suas famílias e 94\% das mães do Grupo Comparação referiram ter um companheiro (casadas ou em união consensual), vivendo em famílias biparentais (Tabela 1).

Quanto às características sociodemográficas das famílias, identificaram-se diferenças estatisticamente significativas na comparação entre os grupos. No que se refere à classificação socioeconômica, verificou-se que a maioria das famílias (58\%) foram classificadas como pertencentes às classes A e B (Grupo Depressão - 38\% e Grupo Comparação - 78\%). Identificou-se o predomínio de famílias (59\%) com renda mensal maior ou igual a três salários mínimos, correspondendo a $46 \%$ das famílias do Grupo Depressão e $72 \%$ das do Grupo Comparação (Tabela 1).

As diferenças significativas identificadas, quando das comparações entre os grupos quanto às condições sociodemográficas, caracterizaram para o Grupo Depressão: menor escolaridade materna, predomínio de constituição familiar monoparental e famílias com classificação socioeconômica menos favorecida. Essas condições foram consideradas como possíveis determinantes sociais que foram considerados na análise de dados, uma vez que na coleta não foi garantida a homogeneidade dos grupos quanto a tais características (Tabela 1). 
Tabela 1. Perfil sociodemográfico e comparações entre as variáveis referentes a características das crianças, das mães e das famílias para os grupos Depressão $(n=50)$ e Comparação $(n=50)$. Ribeirão Preto, 2007 a 2013.

\begin{tabular}{|c|c|c|c|c|c|c|}
\hline & \multicolumn{2}{|c|}{ Grupos } & \multirow{2}{*}{$\begin{array}{c}\text { Total } \\
(n=100)\end{array}$} & \multirow{3}{*}{\multicolumn{2}{|c|}{ IC $(95 \%)$}} & \multirow[b]{3}{*}{ p-valor* } \\
\hline & $\begin{array}{c}\text { Depressão } \\
(n=50)\end{array}$ & $\begin{array}{c}\text { Comparação } \\
(n=50)\end{array}$ & & & & \\
\hline & f (\%) & f (\%) & f (\%) & & & \\
\hline \multicolumn{7}{|l|}{ Crianças } \\
\hline \multicolumn{7}{|l|}{ Idade } \\
\hline 7 a 9 anos & $24(48,00)$ & $26(52,00)$ & $50(50,00)$ & 1,000 & \multirow{2}{*}{$\begin{array}{c}\text { Referência } \\
(0,535 ; 2,572)\end{array}$} & \multirow[t]{2}{*}{0,841} \\
\hline 10 a 12 anos & $26(52,00)$ & $24(48,00)$ & $50(50,00)$ & 1,174 & & \\
\hline \multicolumn{7}{|l|}{ Sexo } \\
\hline Masculino & $24(48,00)$ & $26(52,00)$ & $50(50,00)$ & 1,000 & \multirow{2}{*}{$\begin{array}{c}\text { Referência } \\
(0,535 ; 2,572)\end{array}$} & \multirow[t]{2}{*}{0,841} \\
\hline Feminino & $26(52,00)$ & $24(48,00)$ & $50(50,00)$ & 1,174 & & \\
\hline \multicolumn{7}{|l|}{ Escolaridade } \\
\hline $1^{\circ}$ ao $5^{\circ}$ ano & $29(58,00)$ & $32(64,00)$ & $61(61,00)$ & 1,000 & \multirow{2}{*}{$\begin{array}{c}\text { Referência } \\
(0,575 ; 2,881)\end{array}$} & \multirow[t]{2}{*}{0,682} \\
\hline $6^{\circ}$ ao $8^{\circ}$ ano & $21(42,00)$ & $18(36,00)$ & $39(39,00)$ & 1,287 & & \\
\hline \multicolumn{7}{|l|}{ Mães } \\
\hline \multicolumn{7}{|l|}{ Idade } \\
\hline 25 a 35 anos & $23(46,00)$ & $26(52,00)$ & $49(49,00)$ & 1,000 & Referência & \multirow[t]{2}{*}{0,689} \\
\hline 36 a 45 anos & $27(54,00)$ & $24(48,00)$ & $51(51,00)$ & 1,271 & $(0,580 ; 2,789)$ & \\
\hline \multicolumn{7}{|l|}{ Escolaridade } \\
\hline$\leq 8$ anos & $31(62,00)$ & $13(26,00)$ & $44(44,00)$ & 4,644 & $(1,981 ; 10,883)$ & \multirow[t]{2}{*}{0,001} \\
\hline$>8$ anos & $19(38,00)$ & $37(74,00)$ & $56(56,00)$ & 1,000 & Referência & \\
\hline \multicolumn{7}{|l|}{ Estado Civil } \\
\hline Sem companheiro & $33(66,00)$ & $3(6,00)$ & $36(36,00)$ & $\begin{array}{c}30,41 \\
0\end{array}$ & $(8,242 ; 112,209)$ & \multirow[t]{2}{*}{$<0,001$} \\
\hline Com companheiro & $17(34,00)$ & $47(94,00)$ & $64(64,00)$ & 1,000 & Referência & \\
\hline \multicolumn{7}{|l|}{ Ocupação } \\
\hline Assalariado & $27(54,00)$ & $36(72,00)$ & $63(63,00)$ & 1,000 & Referência & \multirow[t]{2}{*}{0,098} \\
\hline Benefício + Sem trabalho & $23(46,00)$ & $14(28,00)$ & $37(37,00)$ & 2,190 & $(0,954 ; 5,028)$ & \\
\hline \multicolumn{7}{|l|}{ Número de Filhos } \\
\hline 1 a 3 filhos & $40(80,0)$ & $47(94,0)$ & $87(87,00)$ & 1,000 & Referência & \multirow[t]{2}{*}{0,074} \\
\hline 4 a 5 filhos & $10(20,0)$ & $3(6,0)$ & $13(13,00)$ & 3,917 & $(1,008 ; 15,220)$ & \\
\hline \multicolumn{7}{|l|}{ Famílias } \\
\hline \multicolumn{7}{|l|}{ Classe Socioeconômica** } \\
\hline A e B & $19(38,00)$ & $39(78,00)$ & $58(58,00)$ & 1,000 & Referência & \multirow[t]{2}{*}{$<0,001$} \\
\hline C e D & $31(62,00)$ & $11(22,00)$ & $42(42,00)$ & 5,785 & $(2,400 ; 13,942)$ & \\
\hline Renda Mensal*** & & & & & & \\
\hline <3 salários mínimos & $26(52,00)$ & $14(28,00)$ & $40(40,00)$ & 3,156 & $(1,369 ; 7,276)$ & 0,019 \\
\hline$\geq 3$ salários mínimos & $23(46,00)$ & $36(72,00)$ & $59(59,00)$ & 1,000 & Referência & \\
\hline
\end{tabular}

Nota: $\mathrm{f}$ = frequência; $\%$ = Porcentagem; *p-valor referente ao teste Qui-Quadrado / p s 0,05; OR = Odds Ratio; IC (95\%) = Intervalo com 95\% de confiança; **Obtida segundo o Critério de Classificação Econômica Brasil desenvolvido pela Associação Brasileira de Empresas de Pesquisas - ABEP; ${ }^{* * *}$ Calculada em salário mínimo, vigente à época da entrevista.

Verificou-se que todas as mães do Grupo Depressão foram diagnosticadas com Transtorno Depressivo Recorrente, sendo que para a maioria (76\%) o episódio depressivo de maior gravidade foi classificado como moderado e 24\% apresentaram episódio grave (7\% com sintomas psicóticos e 5\% sem sintomas psicóticos). Sete mães (14\%) tiveram pelo menos uma internação psiquiátrica decorrente da sintomatologia depressiva e nove mães (18\%) referiram pelo menos uma tentativa de suicídio nos anos antecedentes à coleta de dados (Tabela 2).

Quanto à recorrência dos episódios depressivos, observou-se que 54\% das mães apresentaram mais de três episódios e que as demais mães apresentaram dois (32\%) ou três (14\%) episódios após o nascimento da criança avaliada. A maioria das mães do Grupo Depressão (78\%) afirmou estar usando antidepressivo no momento da coleta, associado ou não, ao uso de outras medicações psiquiátricas. Ademais, em relação à autopercepção de sua 
condição atual, $68 \%$ das mães desse grupo relataram prejuízos ou recaídas e 32\% a avaliaram como positiva (Tabela 2).

Tabela 2. Perfil clínico das mães do Grupo Depressão em relação à gravidade, à recorrência, ao tratamento da depressão e à percepção materna da condição atual. Ribeirão Preto, 2007 a 2013.

\begin{tabular}{lcc}
\hline Variáveis & & $\mathbf{f ( \% )}$ \\
\hline Diagnóstico* & Episódio Moderado & $38(76,00)$ \\
& Episódio Grave sem Sintomas Psicóticos & $05(10,00)$ \\
& Episódio Grave com Sintomas Psicóticos & $07(14,00)$ \\
Internações & Ausentes & $43(86,00)$ \\
& Presentes & $07(14,00)$ \\
Tentativas de suicídio & Ausentes & $41(82,00)$ \\
& Presentes & $09(18,00)$ \\
Recorrências & 2 episódios & $16(32,00)$ \\
& 3 episódios & $07(14,00)$ \\
Uso atual - antidepressivo & 3 episódios & $27(54,00)$ \\
Percepção da condição atual & Presente & $39(78,00)$ \\
& Ausente & $11(23,00)$
\end{tabular}
Nota: $\mathrm{f}=$ frequência; \% = Porcentagem; *Classificação diagnóstica da depressão, segundo a CID-10.

Dentre as mães com diagnóstico de depressão recorrente (Grupo Depressão), o escore médio relativo à gravidade do transtorno foi de 3,00 (DP=1,53), variando entre escores iguais a 1 (mínimo) e 7 (máximo). Verificou-se que apenas o modelo preditivo para hiperatividade não foi significativo, sendo que a gravidade da depressão materna se mostrou preditora dos demais problemas comportamentais avaliados pelo SDQ total e para as escalas específicas, sintomas emocionais, problemas de conduta e de relacionamento com colegas (Tabela 3).

As análises de regressão linear simples indicaram que a gravidade da depressão materna teve maior poder preditivo para os sintomas emocionais $(F=23,586 ; p<0,001)$, os problemas de relacionamento com colegas $(\mathrm{F}=17,250 ; \mathrm{p}<0,001)$ e o total de dificuldades $(\mathrm{F}=16,853 ; \mathrm{p}$ $<0,001$ ), explicando de $13 \%$ a $18 \%$ da variância dos escores nessas duas escalas e do escore total (Tabela 3).

Destaca-se que, frente às diferenças relativas a características sociodemográficas da amostra entre os grupos, realizou-se ainda uma análise de regressão logística para avaliar o peso dessas variáveis com diferenças significativas para os problemas de comportamento da amostra total de crianças (Tabela 3 ).

Tabela 3. Análises de regressão linear simples considerando como variável preditora a gravidade da depressão materna para os desfechos de problemas comportamentais do SDQ (n = 100). Ribeirão Preto, 2007 a 2013.

\begin{tabular}{lccccccc}
\hline & \multicolumn{6}{c}{ Variável Preditora - Gravidade Depressão Materna } \\
\cline { 2 - 7 } \multicolumn{1}{c}{ Variáveis Desfecho } & $\mathbf{B}$ & $\boldsymbol{\beta}$ & $\mathbf{T}$ & $\mathbf{p}$-valor* & IC (95\%) & $\begin{array}{c}\mathbf{R}^{\mathbf{2}} \\
\text { ajustado }\end{array}$ \\
\hline Total Dificuldades & 1,531 & 0,383 & 4,105 & $<\mathbf{0 , 0 0 1}$ & $0,791-2,271$ & 0,138 \\
Sintomas Emocionais & 0,649 & 0,440 & 4,857 & $<\mathbf{0 , 0 0 1}$ & $0,384-0,914$ & 0,186 \\
Problemas de Conduta & 0,230 & 0,202 & 2,040 & $\mathbf{0 , 0 4 4}$ & $0,006-0,454$ & 0,031 \\
Hiperatividade & 0,271 & 0,154 & 1,545 & 0,126 & $-0,077-0,620$ & 0,014 \\
Relacionamento Colegas & 0,382 & 0,387 & 4,153 & $<\mathbf{0 , 0 0 1}$ & $0,199-0,565$ & 0,141 \\
\hline
\end{tabular}

Nota: ${ }^{*} \mathrm{p}$-valor $=\mathrm{p} \leq 0,05$; IC (95\%) = Intervalo com 95\% de confiança. 
$\mathrm{Na}$ Tabela 4, apresentam-se os indicadores referentes às análises de regressão. Nas análises, identificou-se que nenhuma das variáveis sociodemográficas, consideradas como possíveis determinantes sociais, foram preditoras de mais problemas de comportamento. Tal dado evidencia o papel significativo da depressão materna, avaliada a partir de indicadores cumulativos de gravidade, para os desfechos comportamentais infantis.

Tabela 4. Análise de regressão logística bivariada, tendo como variáveis independentes a escolaridade e o estado civil maternos, a classe socioeconômica e a renda mensal familiar, e como variável dependente as classificações quanto ao comportamento das crianças em Sem Dificuldade ou Com Dificuldade, no SDQ - Escore Total de Dificuldades (Grupo Depressão). Ribeirão Preto, 2007 a 2013.

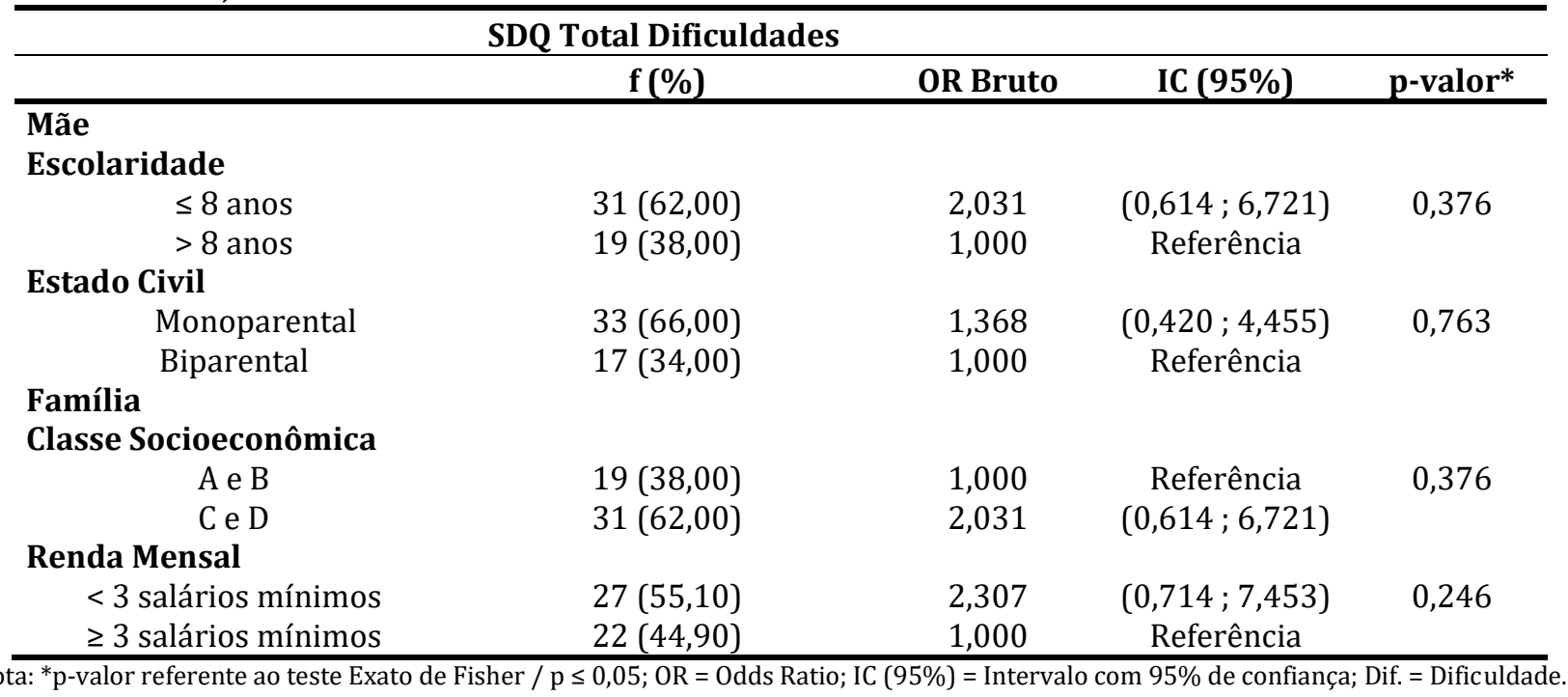

\section{DISCUSSÃO}

Dados relativos às variáveis sociodemográficas evidenciaram que as crianças que convivem com a depressão materna também vivenciam condições de vulnerabilidade relacionadas a esse transtorno. Destaca-se, na pesquisa, a menor escolaridade materna, a monoparentalidade e menos recursos socioeconômicos, fatores que são determinantes sociais que podem compor um cenário familiar de vulnerabilidade para os escolares em questão, tal como sugerido pela OMS ${ }^{11}$.

Estudos empíricos e de revisão colocaram em destaque tais determinantes sociais apresentados pela OMS e os associaram à depressão materna, perfazendo cenários familiares de múltiplos estressores ${ }^{13,14,26,27}$. A presença de múltiplos estressores constitui-se em um modelo explicativo consolidado ${ }^{28}$, o que justificou a inclusão de tais variáveis na compreensão do peso da gravidade da depressão materna para escolares, de modo a se identificar variáveis de vulnerabilidade social, concorrentes relevantes para as famílias.

Por sua vez, neste estudo, essas variáveis sociodemográficas não se mostraram como riscos concorrentes à depressão materna, no sentido de impactar preditivamente desfechos desenvolvimentais infantis negativos, o que reitera o papel de vulnerabilidade dessas condições e não de risco, com aumento de probabilidade de desfechos negativos para os filhos que convivem nessas famílias. Dessa forma, evidencia-se um modelo explicativo que identifica, na presença de diferenças sociodemográficas significativas, enquanto condições de vulnerabilidade social, que a gravidade da depressão materna se configura como a única condição preditora para desfechos de problemas comportamentais na idade escolar, conforme destacado em diferentes estudos empíricos para desfechos desenvolvimentais diversos em escolares que convivem com a depressão materna ${ }^{5-9}$.

Além da presença da variável em si, coloca-se em foco a gravidade da depressão materna recorrente enquanto variável de risco que aumenta a probabilidade de escolares, que convivem 
com esse transtorno, apresentarem problemas comportamentais. Dessa forma, indicadores da gravidade se constituem em características clínicas relevantes e, cumulativamente, preditoras desses problemas, podendo ser utilizados nos diferentes níveis de atenção em saúde, frente à confirmação de episódios depressivos, incluindo as unidades básicas de saúde 4 .

Além do amplo reconhecimento quanto ao impacto da depressão materna recorrente para desfechos negativos e disfuncionais para as crianças ${ }^{13}$, a gravidade desse transtorno clínico também se configurou como indicador, dado que se verificou que, quanto maior número de itens de gravidade presentes, mais problemas comportamentais foram identificados nas crianças.

As dificuldades das crianças foram verificadas para o total de problemas, mas também para sintomas emocionais 5 e problemas de relacionamento, evidenciando o impacto para sintomas internalizantes ${ }^{6,7}$ e externalizantes como os relativos aos problemas de conduta ${ }^{8,9}$. Pondera-se que a identificação de problemas comportamentais dos filhos se deu no presente estudo junto às mães que assumem o papel de principais cuidadoras dos filhos na maioria das culturas.

A inclusão de mães sem sintomas depressivos, nos seis meses anteriores à coleta de dados, foi um cuidado metodológico importante, de forma a favorecer dados menos enviesados pela condição depressiva das mães. A presença de sintomas depressivos se associa a avaliações negativas dos comportamentos dos filhos ${ }^{29}$.

A associação entre gravidade da depressão e maior prejuízo funcional e sofrimento psíquico para a pessoa acometida se amplia, a partir do presente estudo, também para a predição de mais problemas de comportamento em escolares, tendo em vista a maior probabilidade de recorrência dos episódios depressivos e as baixas taxas de recuperação associadas a indicadores de gravidade ${ }^{1}$. Assim, infere-se ainda que a condição relativa ao impacto negativo para as crianças pode se manter ao longo da trajetória de desenvolvimento das crianças, incluindo a convivência com o transtorno materno em outros períodos do ciclo vital.

A gravidade da depressão materna recorrente pôde ser identificada a partir da Entrevista Clínica Estruturada para o DSM-IV20,21, instrumento padrão ouro para a confirmação diagnóstica. Dessa forma, foi possível o acesso a indicadores distintos relativos à gravidade, não disponíveis em outros instrumentos de avaliação, como os de autorrelato e de rastreamento.

0 acesso a indicadores de gravidade distintos e a construção de uma codificação e pontuação desses indicadores permitiram a construção de um escore de gravidade com itens cumulativos que ampliam a forma de se considerar qualitativamente a variável depressão, enquanto preditora de problemas para os filhos.

A utilização de instrumentos diagnósticos, de maneira geral, demanda maior tempo em comparação aos instrumentos de rastreamento. Nesse sentido, o uso de um instrumento de rastreamento da avaliação sistemática por meio de um conjunto de indicadores clínicos de gravidade pode favorecer uma caracterização mais rápida e precisa. Vislumbra-se, assim, que a lista de indicadores de gravidade aqui construída poderá ser utilizada como recurso associado aos indicadores de rastreamento para avaliar a gravidade da depressão materna.

\section{CONCLUSÃo}

A sistematização de indicadores clínicos associados à gravidade da depressão recorrente em mulheres/mães de escolares favoreceu uma compreensão ampliada desse transtorno enquanto preditor de problemas comportamentais no geral e de sintomas internalizantes e externalizantes para escolares.

Os itens elencados mostraram-se relevantes para a identificação do peso da gravidade para os desfechos infantis e podem, pois, se configurar como recursos complementares para a avaliação de gravidade quando do uso de instrumentos de rastreamento. Nesse sentido, o modelo testado quanto ao impacto negativo da gravidade da depressão materna para 
problemas de comportamento nos filhos, em idade escolar, se revelou também como uma possibilidade de avanço na compreensão de preditores para o desenvolvimento infantil, na presença de importantes determinantes sociais associados às condições sociodemográficas das famílias.

Considera-se como limites do estudo, o delineamento transversal e a amostra de conveniência acessada em serviços clínicos específicos, o que limita a generalização dos achados, sugerindo-se futuros estudos longitudinais.

Apesar disto, configura-se como principal contribuição do estudo a verificação do impacto diferenciado da gravidade da depressão, avaliada por meio de indicadores clínicos específicos, os quais, pela facilidade de aplicação, podem ser utilizados como complementares na avaliação clínica sistemática de rastreamento da depressão em diferentes níveis de atenção em saúde, ampliando assim a qualidade das avaliações, o que por sua vez favorece os cuidados com a saúde mental materna e infantil.

\section{REFERÊNCIAS}

1. American Psychiatric Association. Manual Diagnóstico e Estatístico de Transtornos Mentais - DSM-5. 9ed. Porto Alegre: Artmed Editora; 2014.

2. World Health Organization. Depression and other commom mental health [Internet]. Geneva: WHO; 2017 [citado em 16 abr 2019]. Disponível em: https://apps.who.int/iris/bitstream/handle/10665/254610/WHO-MSD-MER-2017.2-

eng.pdf;jsessionid $=9862 \mathrm{C} 65 \mathrm{~A} 30 \mathrm{FA4A7808AB011308217674}$ ? sequence $=1$

3. World Health Organization. Depression [Internet]. Geneva: WHO; 2018 [citado em 16 abr 2019]. Disponível em: https://www.who.int/en/news-room/fact-sheets/detail/depression

4. Molina MRAL, Wiener CD, Branco JC, Jansen K, Souza LDMD, Tomasi E, Pinheiro RT. Prevalência de depressão em usuários de unidades de atenção primária. Rev Psiquiatr Clín. [Internet]. 2012 [citado em 16 abr 2019]; 39(6):194-7. Disponível em: http://www.scielo.br/pdf/rpc/v39n6/03.pdf DOI: http://dx.doi.org/10.1590/S0101-60832012000600003

5. Cardoso TSG, Siquara GM, Freitas PM. Relações entre depressão materna e problemas de comportamento em crianças. Psicol Argum. [Internet]. 2014 [citado em 16 abr 2019]; 32(79):131-41. DOI: http://dx.doi.org/10.7213/psicol.argum.32.079.A008

6. Hser Y, Lanza I, Li L, Kahn E, Evans E, Schulte M. Maternal mental health and children's internalizing and externalizing behaviors: beyond maternal substance use disorders. J Child Fam Stud. [Internet]. 2015 [citado em 16 abr 2019]; 24:638-48. Disponível em: https://www.ncbi.nlm.nih.gov/pmc/articles/PMC4349431/pdf/nihms543556.pdf DOI: http://dx.doi.org/10.1007/s10826-013-9874-3

7. Jacobs RH, Talati A, Wickramaratne P, Warner V. The influence of paternal and maternal major depressive disorder on offspring psychiatric disorders. J Child Fam Stud. [Internet]. 2015 [citado em 16 abr 2019]; 24(8):2345-51. Disponível em: https://www.ncbi.nlm.nih.gov/pmc/articles/PMC4706755/pdf/nihms625139.pdf DOI: http://dx.doi.org/10.1007/s10826-014-0037-y

8. Van Doorn MME, Kuijpers RCWM, Lichtwarck-Aschoff A, Bodden D, Jansen M, Gramic I. Does motherchild interaction mediate the relation between maternal depressive symptoms and children's mental health problems? J Child Fam Stud. [Internet]. 2016 [citado em 16 abr 2019]; 25:1257-68. Disponível em: https://www.ncbi.nlm.nih.gov/pmc/articles/PMC4779455/pdf/10826_2015_Article_309.pdf DOI: http://dx.doi.org/10.1007/s10826-015-0309-1

9. Yan N, Zhou N, Ansari A. Maternal depression and children's cognitive and socio-emotional development at first grade: the moderating role of classroom emotional climate J Child Fam Stud. [Internet]. 2016 [citado em 16 abr 2019]; 25:1247-56. Disponível em: https://link.springer.com/content/pdf/10.1007\%2Fs10826-015-0301-9.pdf DOI: http://dx.doi.org/10.1007/s10826-015-0301-9

10. Boyd RC, Waanders C. Protective factors for depression among African American children of predominantly low-income mothers with depression. J Child Fam Stud. [Internet]. 2013 [citado em 16 $\mathrm{abr}$

2019];

22:85-95.

Disponível

em: 
https://www.ncbi.nlm.nih.gov/pmc/articles/PMC4196212/pdf/nihms-628256.pdf

DOI: http://dx.doi.org/10.1007/s10826-012-9588-y

11. World Health Organization. Social determinants of mental health [Internet]. Geneva: WHO; 2014 [citado em 16 abr 2019]. Disponível em: http://www.who.int/mental_health/publications/gulbenkian_ paper_social_determinants_of_mental_health/en/

12. Sweeney S, MacBeth M. The effects of paternal depression on child and adolescent outcomes: a systematic review. J Affect Disord. [Internet]. 2016 [citado em 16 abr 2019]; 15(205):44-59. Disponível em:

https://reader.elsevier.com/reader/sd/pii/S0165032715312404?token=346E3F6C0928F805366A98 5B7183A9F29D9A18652A17A905581203636920B9164E9774DEEA9567AC2BC32272E7DB4140.

DOI: http://dx.doi.org/10.1016/j.jad.2016.05.073

13. Goodman SH, Rouse MH, Connel AM, Broth MR, Hall CM, Heyward D. Maternal depression and child psychopathology: a meta-analytic review. Clin Child Fam Psychol Rev. [Internet]. 2011 [citado em 16 abr 2019]; 14(1):1-27. Disponível em: https://link.springer.com/content/pdf/10.1007\%2Fs10567010-0080-1.pdf. DOI: http://dx.doi.org/10.1007/s10567-010-0080-1

14. Pizeta FA, Silva TBF, Cartafina MIB, Loureiro SR. Depressão materna e riscos para o comportamento e a saúde mental das crianças: uma revisão. Estud Psicol. (Natal) [Internet]. 2013 [citado em 16 abr 2019]; 18(3):429-37. Disponível em: http://www.scielo.br/pdf/epsic/v18n3/03.pdf DOI: http://dx.doi.org/10.1590/S1413-294X2013000300003

15. Organização Mundial de Saúde. Classificação de transtornos mentais e de comportamento da CID10: descrições clínicas e diretrizes diagnósticas. Porto Alegre: Artes Médicas; 2014.

16. Schmidt MI, Duncan BB, Azevedo e Silva G, Menezes AM, Monteiro CA, Barreto SM, et al. Chronic noncommunicable diseases in Brazil: burden and current challenges. Lancet [Internet]. 2011 [citado em 16 abr 2019]; 377:1949-61. Disponível em: https://reader.elsevier.com/reader/sd/pii/S0140673611601359?token=73D55812D237719577E92 FE94CDC158C655408C074F653A7D51F4B448F847878031FDC7BD4C89E631176D62AA3800017 DOI: http://dx.doi.org/10.1016/S0140-6736(11)60135-9

17. Spitzer R, Kroenke K, Williams J. Validation and utility of a self-report Version of PRIME-MD: the PHQ Primary Care Study. J Am Med Assoc. [Internet]. 1999 [citado em 16 abr 2019]; 282(18):1737-44. Disponível em: https://jamanetwork.com/journals/jama/fullarticle/192080 DOI: http://dx.doi.org/10.1001/jama.282.18.1737

18. Kroenke K, Spitzer RL, Williams JB. The PHQ-9: validity of a brief depression severity measure. J Gen Intern Med. [Internet]. 2001 [citado em 16 abr 2019]; 16(9):606-13. Disponível em: https://www.ncbi.nlm.nih.gov/pmc/articles/PMC1495268/pdf/jgi_01114.pdf DOI: http://dx.doi.org/10.1046/j.1525-1497.2001.016009606.x

19. Osório FL, Mendes AV, Crippa JA, Loureiro SR. Study of the discriminative validity of the PHQ-9 and PHQ-2 in a sample of Brazilian women in the context of primary health care. Perspect Psychiatr Care [Internet]. 2009 [citado em 16 abr 2019]; 45(3):216-27. Disponível em: https://onlinelibrary.wiley.com/doi/epdf/10.1111/j.1744-6163.2009.00224.x DOI: http://dx.doi.org/10.1111/j.1744-6163.2009.00224.x

20. Del-Ben CM. Estudo da confiabilidade do diagnóstico psiquiátrico obtido através da entrevista clínica estruturada para o DSM-III-R (SCID) em serviço ambulatorial de um hospital escola. [dissertação]. Ribeirão Preto, SP: Faculdade de Medicina de Ribeirão Preto, Universidade de São Paulo; 1995.

21. Del-Ben CM, Vilela JAA, Crippa JAS, Hallak JEC, Labate CM, Zuardi AW. Confiabilidade da Entrevista Clínica Estruturada para DSM-IV - versão clínica traduzida para o português. Rev Bras Psiquiatr. [Internet]. 2001 [citado em 16 abr 2019]; 3(23):156-9. Disponível em: http://www.scielo.br/pdf/rbp/v23n3/a08v23n3.pdf DOI: http://dx.doi.org/10.1590/S151644462001000300008

22. Goodman R. The strengths and difficulties questionnaire: a research note. J Child Psychol Psychiatry [Internet]. 1997 [citado em 16 abr 2019]; 38(5):581-6. Disponível em: https://onlinelibrary.wiley.com/doi/epdf/10.1111/j.1469-7610.1997.tb01545.x DOI: http://dx.doi.org/10.1111/j.1469-7610.1997.tb01545.x

23. Fleitlich BW, Cortázar PG, Goodman R. Questionário de Capacidades e Dificuldades (SDQ). Infanto Rev Neuropsiquiatr Infânc Adolesc. [Internet]. 2000 [citado em 16 abr 2019]; 1(8):44-50. Disponível em: https://pesquisa.bvsalud.org/portal/resource/pt/lil-275954 
24. Woerner W, Fleitlich-Bilyk B, Martinussen R, Fletcher J, Cucchiaro G, Dalgalarrondo P, et al. The Strengths and Difficulties Questionnaire overseas: evaluations e applications of the SDQ beyond Europe. Eur Child Adolesc Psychiatry [Internet]. 2004 [citado em 16 abr 2019]; 13(Suppl. 2):II47-II54. Disponível em: https://link.springer.com/content/pdf/10.1007\%2Fs00787-004-2008-0.pdf. DOI: http://dx.doi.org/10.1007/s00787-004-2008-0

25. Maroco J Análise estatística com o SPSS. 6a ed. Pero Pinheiro, Portugal: Ed. Reportnumber; 2014.

26. Barker ED, Copeland W, Maughan B, Jaffee SR, Uher R. The relative impact of maternal depression and associated risk factors on offspring psychopathology. Br J Psychiatr. [Internet]. 2012 [citado em 05 de jan 2020]; 200(2):124-9. Disponível em: https://www.cambridge.org/core/services/aopcambridge-

core/content/view/2C2DBAD78CB2763623790D8D85F8518F/S0007125000257590a.pdf/relative_i mpact_of_maternal_depression_and_associated_risk_factors_on_offspring_psychopathology.pdf DOI: http://dx.doi.org/10.1192/bjp.bp.111.092346

27. Bouvette-Turcot AA, Unternaehrer E, Gaudreau H, Lydon JE, Steiner M, Meaney MJ. The joint contribution of maternal history of early adversity and adulthood depression to socioeconomic status and potential relevance for offspring development. J Affect Disord. [Internet]. 2017 [citado em 05 de jan 2020]; 207:26-31. Disponível em: https://reader.elsevier.com/reader/sd/pii/S0165032716304864?token=9BC36B833AE0CDD06046D 4D630C87BCA49B6DEE44DBE12B6EA787FDAACB05C220002C021D03FC8DE03007CD2BB8AC9A1 DOI: http://dx.doi.org/10.1016/jjad.2016.08.012

28. Evans GW, Li D, Whipple SS. Cumulative risk and child development. Psychol Bull. [Internet]. 2013 [citado em 05 de jan 2020]; 139(6):1342-96. Disponível em: https://psycnet.apa.org/fulltext/201312151-001.pdf. DOI: http://dx.doi.org/10.1037/a0031808

29. Callender KA, Olson SL, Choe DE, Sameroff AJ. The effects of parental depressive symptoms, appraisals, and physical punishment on later child externalizing behavior. J Abnorm Child Psychol. [Internet]. 2012 [citado em 05 de jan 2020]; 40(3):471-83. Disponível em: $\quad$ https://link.springer.com/content/pdf/10.1007\%2Fs10802-011-9572-9.pdf $\quad$ DOI: http://dx.doi.org/10.1007/s10802-011-9572-9

\section{CONTRIBUIÇÕES}

Fernanda Aguiar Pizeta, Ana Paula Casagrande Silva-Rodrigues contribuíram na concepção, delineamento, coleta de dados, análise e interpretação dos dados, revisão e redação. Sonia Regina Loureiro participou na concepção, delineamento, análise e interpretação dos dados, revisão e redação.

\section{Como citar este artigo (Vancouver)}

Pizeta FA, Silva-Rodrigues APC, Loureiro SR. Indicadores de gravidade da depressão materna: impacto para problemas comportamentais de escolares. REFACS [Internet]. 2020 [citado em inserir dia, mês e ano de acesso]; 8(Supl. 1):524-536. Disponível em: inserir link de acesso. DOI: inserir link do DOI.

\section{Como citar este artigo (ABNT)}

PIZETA, F. A.; SILVA-RODRIGUES, A. P. C.; LOUREIRO, S. R. Indicadores de gravidade da depressão materna: impacto para problemas comportamentais de escolares. REFACS, Uberaba, MG, v. 8, p. 524-536, 2020. Supl. 1. Disponível em: inserir link de acesso. Acesso em: inserir dia, mês e ano de acesso. DOI: inserir link do DOI.

\section{Como citar este artigo (APA)}

Pizeta, F.A., Silva-Rodrigues, A.P.C., \& Loureiro, S.R. (2020). Indicadores de gravidade da depressão materna: impacto para problemas comportamentais de escolares. REFACS, 8(Supl. 1), 524-536. Recuperado em: inserir dia, mês e ano de acesso de inserir link de acesso. DOI: inserir link do DOI. 